\title{
L-band R-EDFA/Raman hybrid amplifier with enhanced higher-order pumping scheme utilizing stimulated Raman scattering.
}

\begin{abstract}
An enhanced pump delivery scheme for L-band remote erbium-doped fiber/Raman (REDFA/Raman) hybrid amplifier utilizing stimulated Raman scattering (SRS) is demonstrated. The technique benefits from the use of higher-order pumps that are realized by generating Cband SRS and ultra-long Raman fiber laser (ULRFL). A section of passive erbium-doped fiber is deployed at the end of the transmission span to exploit the ULRFL pump for additional amplification. Gain clamping effect is observed at the R-EDFA and passive EDF due to saturation from the higher-order pumps. Tuning the pump wavelengths to a region away from the amplification bandwidth could remove the gain-clamping effect and produce maximum gain performance.
\end{abstract}

Keyword: Amplifiers; Erbium; Stimulated Raman scattering; Fiber laser 